\title{
Lattice Quark Propagator in Landau and Laplacian Gauges
}

\author{
Patrick O. Bowman ${ }^{\mathrm{a} *}$, Urs M. Heller ${ }^{\mathrm{a}}$ and Anthony G. Williams ${ }^{\mathrm{b}}$

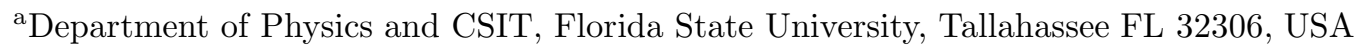 \\ ${ }^{\mathrm{b}} \mathrm{CSSM}$ and Department of Physics and Mathematical Physics, Adelaide University, Australia 5005
}

We present results for the lattice quark propagator in both Landau and Laplacian gauges using standard and improved staggered quark actions. The standard Kogut-Susskind action has errors of $\mathcal{O}\left(a^{2}\right)$ while the improved "Asqtad" action has $\mathcal{O}\left(a^{4}\right), \mathcal{O}\left(a^{2} g^{2}\right)$ errors. This improvement is seen in the quark propagator. We demonstrate the application of tree-level corrections to these actions and see that Landau and Laplacian gauges produce very similar results.

\section{Introduction}

The quark propagator is a fundamental quantity of QCD. Though gauge dependent, it manifestly displays dynamical chiral symmetry breaking, contains the chiral condensate and $\Lambda_{\mathrm{QCD}}$ and has been used to compute the running quark mass (see Ref. [1] and references therein). Some model hadron calculations rely on ansätze for the quark propagator, yet on the lattice we have the opportunity to study it in a direct, nonperturbative fashion. Quark propagator studies can be complicated, however, by strong lattice artefacts [2,33.

We are required to fix a gauge and we choose the Landau and the Laplacian gauges [4]. We use Wilson glue at $\beta=5.85(a \simeq 0.125 \mathrm{fm})$ on a $16^{3} \times$ 32 lattice and six quark masses from $a m=0.075$ down to 0.0125 (115 to $19 \mathrm{MeV}$ ). Calculations were done on 80 configurations.

In the (Euclidean) continuum, Lorentz invariance allows us to decompose the full propagator into Dirac vector and scalar pieces

$$
S^{-1}\left(p^{2}\right)=Z^{-1}\left(p^{2}\right)\left[i \gamma \cdot p+M\left(p^{2}\right)\right] .
$$

Asymptotic freedom means that, as $p^{2} \rightarrow \infty$, $S^{-1}\left(p^{2}\right) \rightarrow i \gamma \cdot p+m$, (the free propagator) where $m$ is the bare quark mass.

We use the "Asqtad" quark action [5], a fatlink Staggered action using three-link, five-link and seven-link staples to minimise flavour changing interactions along with the three-link Naik

\footnotetext{
*Presented by POB.
}

term and planar five-link Lepage term. The coefficients are tadpole improved and tuned to remove all tree-level $\mathcal{O}\left(a^{2}\right)$ errors. This action was motivated by the desire to improve flavour symmetry, but has also been reported to have good rotational properties.

From consideration of the tree-level forms of our two actions, we define the new momentum variables $q_{\mu} \equiv \sin \left(p_{\mu}\right)$ and $q_{\mu} \equiv \sin \left(p_{\mu}\right)[1+$ $\left.\frac{1}{6} \sin ^{2}\left(p_{\mu}\right)\right]$ for the KS and Asqtad actions respectively. By considering the propagator as a function of $q_{\mu}$, we ensure that the lattice quark propagator has the correct tree-level form and hopefully better approximates its continuum behaviour. This is the same philosphy that has been used in studies of the gluon propagator (see Ref. [6] and references therein). See also footnote 6 in Ref. [1].

\section{Laplacian Gauge}

Laplacian gauge is a nonlinear gauge fixing that respects rotational invariance yet is free of Gribov ambiguities. Although it is difficult to understand perturbatively, it is equivalent to Landau gauge in the asymptotic region. It is also computationally cheaper then Landau gauge. There is, however, more than one way of obtaining such a gauge fixing in $\mathrm{SU}(\mathrm{N})$. The three implementations of Laplacian gauge fixing discussed are

1. $\partial^{2}(\mathrm{I})$ gauge (QR decomposition), used in Ref. [7]. 
2. $\partial^{2}$ (II) gauge, where the complex $3 \times 3$ matrix is projected onto $\mathrm{SU}(3)$ by maximising its trace. This will be discussed in more detail in an upcoming work on the gluon propagator [8].

3. $\partial^{2}$ (III) gauge (Polar decomposition), the original prescription described in Ref. [四].

The gauge transformations employed in Laplacian gauge fixing are constructed from the lowest eigenvectors of the covariant lattice Laplacian operator. The three implementations discussed differ in the way that the gauge transformation is constructed from the above eigenvectors. We can think of each projection method as defining its own Laplacian gauge. In all cases the resulting gauge is unambiguous for all configurations except a set of measure zero.

\section{Results}

We investigated the applicaton of tree-level correction to the quark propagator by comparing the $Z$ functions using $p$ and $q$. We saw that hypercubic artefacts were small when we used $p$ and they vanished almost entirely when using $q$. It is less clear which momentum variable should be used for the mass function, because at tree-level it is not multiplied by the momentum, but for consistency we use $q$ here as well. In the case of the mass function, the choice of momentum will actually make little difference to our results.

In Fig. 1 the mass function is plotted, in Landau gauge, for both actions with quark mass $m a=0.05$. We see that the KS action gives a much larger value for $\mathrm{M}(0)$ than the Asqtad action and is slower to approach asymptotic behaviour. The Asqtad action also shows slightly better rotational symmetry.

The Asqtad action displays clearly better rotational symmetry in the quark $Z$ function and, curiously, improved infrared behaviour as well. The Asqtad action also displays a better approach to asymptopia, approaching one in the ultraviolet. Furthermore, the relative improvement increases as the quark mass decreases. Comparing the mass function for the two actions at $m a=0.0125$, the lowest mass studied here, the low quark mass has
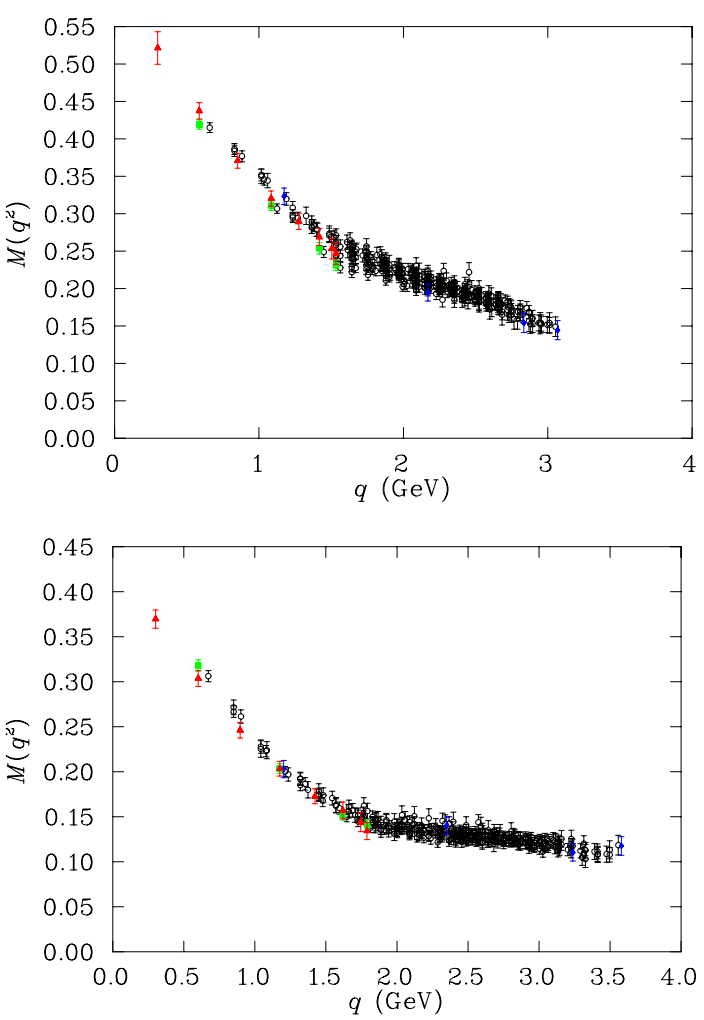

Figure 1. Mass function for quark mass $m a=$ 0.05 ( $m \simeq 77 \mathrm{MeV}$ ), KS action (top) and Asqtad action (bottom) in Landau gauge.

introduced less noise into the propagator with the Asqtad action than with the KS action.

Fig. 2 (top) shows the $Z$ function for the Asqtad action in Landau and $\partial^{2}(\mathrm{I})$ gauges. Data has been cylinder cut [6] for easier comparison. They are in excellent agreement in the ultraviolet but differ significantly in the infrared. There appears to be some slight difference in the $Z$ function between $\partial^{2}(\mathrm{I})$ and $\partial^{2}(\mathrm{II})$ gauges.

Fig. 2 (bottom) shows the mass function for the Asqtad action in Landau and $\partial^{2}(\mathrm{I})$ gauges. The two mass functions agree in the ultraviolet and in the infrared, but the Landau gauge mass function sits slightly higher in the intermediate region. The mass functions are nearly identical in $\partial^{2}(\mathrm{I})$ and $\partial^{2}(\mathrm{II})$ gauges. We have also found that Landau gauge gives slightly less anisotropy at this lattice spacing. 

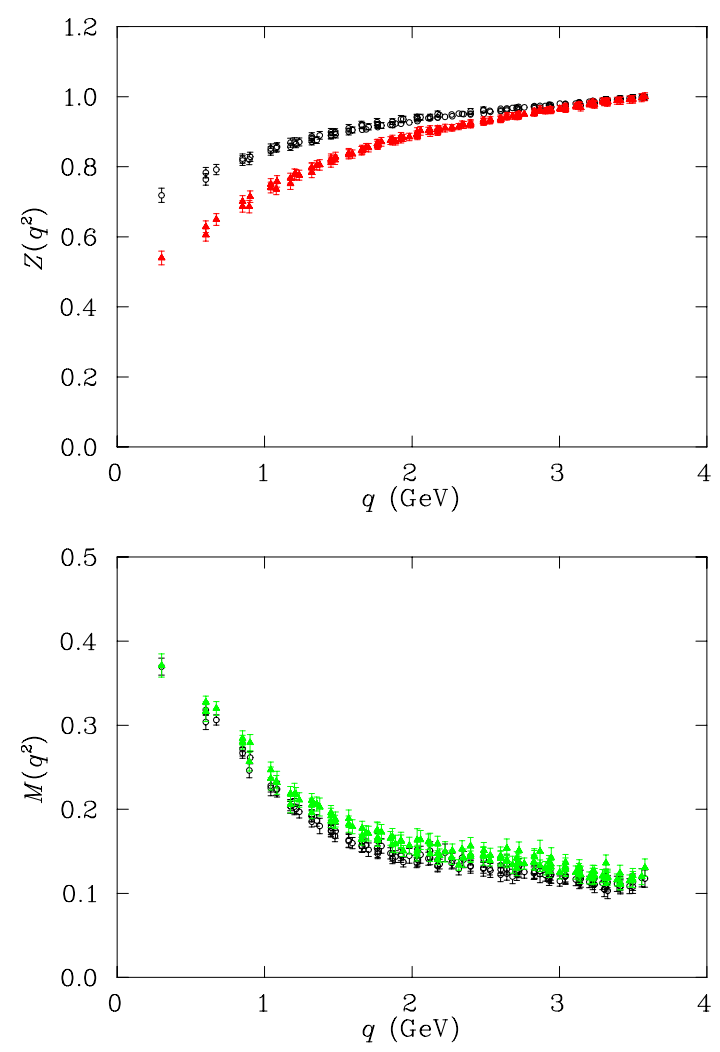

Figure 2. Gauge dependence of the quark Z (top) and mass (bottom) functions for the Asqtad action $(m a=0.05)$. Points marked with open circles are in Landau gauge and solid triangles are in $\partial^{2}(\mathrm{I})$ gauge. Data has been cylinder cut.

$\partial^{2}($ III) performs very poorly. We found that many of the matrices had vanishingly small determinants (compared to numerical precision), which destroyed the projection onto SU(3). Problems with $\partial^{2}$ (III) have also been seen in the gluon propagator \&].

A fit to each of the mass functions, including the chiral limit, was performed using the ansatz

$$
M(p)=\frac{c \Lambda^{1+2 \alpha}}{p^{2 \alpha}+\Lambda^{2 \alpha}}+m_{0}
$$

which is a generalisation of the one used in Ref. [2]. $\alpha>1$ is increasingly favoured as the quark mass approaches zero. We show here, in Fig. 3, one fit for the mass function in the chiral limit.

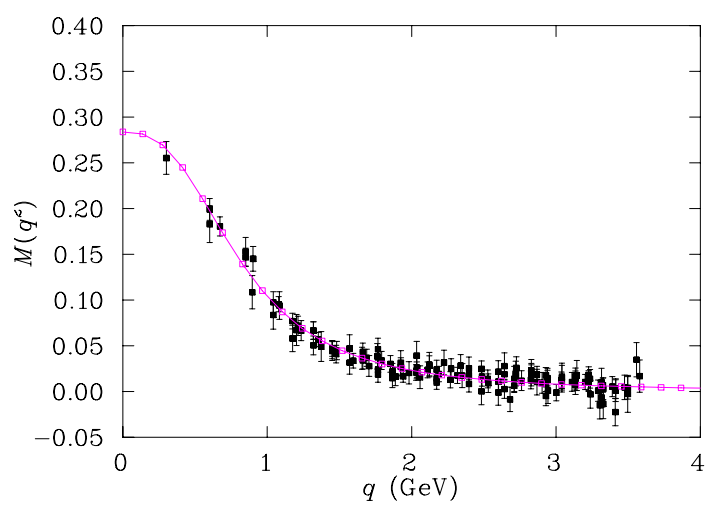

Figure 3. Mass function extrapolated to the chiral limit. Errors are Jack-knife. Fit parameters are $\mathrm{c}=0.030(4), \Lambda=870(60) \mathrm{MeV}, m_{0}=0.0, \alpha=$ $1.52(23), \chi^{2} / \operatorname{dof}=0.49$.

As we have simulated on only one lattice, it remains to do a thorough examination of discretisation and finite volume effects. In forthcoming work we will also examine the gluon propagator in the Laplacian gauges.

\section{Acknowledgments}

The authors wish to thank Derek Leinweber and Jonivar Skullerud for useful discussions.

\section{REFERENCES}

1. D. Becirevic et al., Phys. Rev. D 61, 114507 (2000).

2. J-I. Skullerud and A.G. Williams, Phys. Rev. D 63, 054508 (2001).

3. J. Skullerud et al., Phys. Rev. D 64, 074508 (2001).

4. J.C. Vink and U-J. Wiese, Phys. Lett. B 289, 122 (1992).

5. K. Orginos, D. Toussaint and R.L. Sugar, Phys. Rev. D 60, 054503 (1999).

6. F.D.R. Bonnet et al., Phys. Rev. D 64, 034501 (2001).

7. C. Alexandrou, Ph. de Forcrand and E. Follana, Phys. Rev. D 63, 094504 (2001).

8. P.O. Bowman, U.M. Heller, D.B. Leinweber and A.G. Williams, Work in progress. 